\title{
Prevalência de sinais radiográficos de impacto femoroacetabular em indivíduos assintomáticos e não atletas
}

\section{Prevalence of Radiographic Signs of Femoroacetabular Impingement in Asymptomatic Patients and Non-Athletes}

\author{
André Sousa Garcia ${ }^{1}$ Murilo Gobetti ${ }^{1}$ Anderson Yutaka Tatei ${ }^{1} \quad$ Guilherme Guadagnini Falótico ${ }^{1}$ \\ Gustavo Gonçalves Arliani ${ }^{1}$ Eduardo Barros Puertas ${ }^{1}$ \\ ${ }^{1}$ Escola Paulista de Medicina (EPM), Departamento de Ortopedia e \\ Address for correspondence André Sousa Garcia, Departamento de \\ Traumatologia, Universidade Federal de São Paulo (UNIFESP), \\ São Paulo, SP, Brasil \\ Ortopedia e Traumatologia, Escola Paulista de Medicina (EPM), \\ Universidade Federal de São Paulo (UNIFESP), São Paulo, \\ Rev Bras Ortop 2019;54:60-63. \\ SP 04040-003, Brasil (e-mail: andre_sgarcia@hotmail.com).
}

\section{Resumo}

\section{Palavras-chave}

- impacto femoroacetabular

- quadril

- lesões no quadril

Objetivo O impacto femoroacetabular foi descrito como uma variação anatômica do fêmur proximal e/ou da borda acetabular, causa impacto na articulação do quadril. Uma parcela da população assintomática quanto ao quadril pode apresentar alterações radiográficas de impacto femoroacetabular. O objetivo do estudo é avaliar a prevalência desses sinais em indivíduos do sexo masculino assintomáticos e sedentários. Métodos Estudo clínico, observacional, primário, transversal, controlado. Foram selecionados 32 voluntários masculinos, de 18 a 40 anos, assintomáticos quanto ao quadril, sedentários, atendidos em um Pronto-Socorro de Ortopedia de Hospital Universitário. Todos fizeram radiografias anteroposteriores da pelve padronizadas. Foram analisadas as medidas de ângulo alfa, índice de retroversão, sinal da espinha isquiática e sinal da parede posterior.

Resultados A média de idade foi de 29 anos (18-40). A prevalência de sinais radiográficos de impacto femoroacetabular com o uso do ângulo alfa de 67o foi de $53,1 \%$; com o ângulo alfa de 820 , essa prevalência foi de $31,2 \%$. A média do ângulo alfa foi de 67 o $(52,4-88,20), 35,9 \%$ dos quadris foram classificados como limítrofes e $6,3 \%$ como patológicos. A média do ângulo alfa para o lado direito foi de 67,50 (52,5-88,20) e para o esquerdo, 66,6 o $(53,1-86,90)$. O índice de retroversão médio foi de 0,048 (lado direito - 0,044 e lado esquerdo - 0,052). O sinal da espinha foi positivo em $15,6 \%$ e da parede posterior em $20,3 \%$.
\end{abstract}

\footnotetext{
Trabalho desenvolvido no Escola Paulista de Medicina (EPM), Departamento de Ortopedia e Traumatologia, Universidade Federal de São Paulo (Unifesp), São Paulo, SP, Brasil. Publicado originalmente por Elsevier Editora Ltda. (c) 2018 Sociedade Brasileira de Ortopedia e Traumatologia.

(1) André Sousa Garcia's ORCID is https://orcid.org/0000-0002-93392853.
}

received

September 20, 2017

accepted

November 6, 2017

published online

March 1, 2018
DOI https://doi.org/

10.1016/j.rbo.2017.11.004. ISSN 0102-3616.
Copyright $\odot 2019$ by Sociedade Brasileira License terms de Ortopedia e Traumatologia. Published by Thieme Revnter Publicações Ltda, Rio de Janeiro, Brazil 


\section{Abstract \\ Keywords \\ - femoroacetabular impingement \\ - hip \\ - hip injuries}

Conclusão O presente estudo demonstrou que a prevalência de sinais radiográficos numa população de homens adultos, assintomáticos e sedentários foi elevada (31,2\%). O real significado clínico desse achado ainda carece de novos estudos.

Objective Femoroacetabular impingement has been described as an anatomical variation of the proximal femur and/or acetabular rim, impinging the hip joint. A portion of the population asymptomatic in the hip may present radiographic changes from femoroacetabular impingement. The aim of the present study was to evaluate the prevalence of these signs in asymptomatic and sedentary males.

Methods This was a clinical, observational, primary, cross-sectional, controlled study. A total of 32 male volunteers aged between 18 and 40 years, asymptomatic in the hip and sedentary, were selected from a university hospital orthopedic emergency room. All patients underwent standard anteroposterior pelvic radiographs. The measurements of the alpha angle, the retroversion index, the ischial spine signal, and the posterior wall sign were analyzed.

Results The mean age was 29 years ( $18-40$ years old). The prevalence of radiographic signs of femoroacetabular impingement using an alpha angle of $67^{\circ}$ was of $53.1 \%$; with an alpha angle of $82^{\circ}$, it was of $31.2 \%$. The mean alpha angle was $67^{\circ}\left(52.4-88.2^{\circ}\right)$, with $35.9 \%$ of the hips classified as borderline and $6.3 \%$ as pathological. The mean alpha angle for the right side was $67.5^{\circ}\left(52.5-88.2^{\circ}\right)$, and, for the left, it was $66.6^{\circ}(53.1-$ $\left.86.9^{\circ}\right)$. The mean retroversion index was 0.048 (right side: 0.044 ; left side: 0.052 ). The spine signal was positive in $15.6 \%$, and the posterior wall sign, in $20.3 \%$ of the cases. Conclusion This study showed that the prevalence of radiographic signs in a population of asymptomatic and sedentary adult men was high (31.2\%). New studies are required to explian the actual clinical significance of this finding.

\section{Introdução}

O impacto femoracetabular (IFA) foi descrito por Ganz et al. apud Volpon1 como devido a uma variação anatômica do fêmur proximal (transição colo-cabeça) e/ou borda do acetábulo, que causa impacto na articulação do quadril, principalmente durante os extremos de movimento, o que pode levar a lesões labrais e da cartilagem articular. ${ }^{1-4}$

Classicamente, podemos identificar três tipos básicos de impacto descritos: tipo cam (deformidade na transição colocabeça femoral), tipo pincer (aumento na cobertura acetabular) e o tipo misto (alterações combinadas).,

Ocorre, porém, que uma parcela da população assintomática quanto ao quadril pode apresentar alterações radiográficas de impacto femoroacetabular e seu significado clínico ainda não é totalmente elucidado. ${ }^{2}$ Na literatura nacional são escassos os trabalhos sobre o assunto. Dessa forma, o objetivo do presente estudo é avaliar a prevalência de sinais radiográficos de impacto femoroacetabular em indivíduos do sexo masculino assintomáticos quanto ao quadril e à pelve atendidos no pronto-socorro de um hospital universitário.

\section{Material e Métodos}

Estudo clínico, observacional, primário, transversal, controlado desenvolvido em centro único e aprovado no comitê de ética da instituição em que foi feito sob o número CAAE
54363516.4.0000.5505. Para orientar a metodologia, foi usado o check-list Strobe (11). Foram selecionados 32 voluntários masculinos, de 18 a 40 anos, examinados no ProntoSocorro de Ortopedia de um hospital universitário, assintomáticos quanto aos quadris e sedentários.

Foram usados como critérios de não inclusão a presença de alterações radiográficas, tais como osteoatrite do quadril, osteonecrose da cabeça femoral, sequela de doenças do quadril da infância, sequela de fraturas da pelve, acetábulo ou extremidade proximal do fêmur, bem como radiografia com técnica inadequada.

Nas avaliações, foram registradas as seguintes informações: idade atual e presença da radiografia digital anteroposterior da pelve padronizada (paciente em decúbito dorsal, membros inferiores com rotação interna de 15。, ampola localizada a $120 \mathrm{~cm}$ do paciente com raio centrado $1 \mathrm{~cm}$ acima da sínfise púbica). ${ }^{7}$

\section{Avaliação Radiográfica}

Foram avaliados os seguintes parâmetros radiográficos: ângulo alfa, índice de retroversão, sinal da espinha isquiática e sinal da parede posterior. A análise radiográfica foi feita por um ortopedista e um radiologista, ambos com mais de cinco anos de experiência na área musculoesquelética e cegados quanto ao grupo de origem dos participantes, foi usado para as aferições o software Horos. 
Os valores de ângulo alfa foram avaliados de forma quantitativa e depois categorizados em normal (abaixo de 69॰), limítrofe (entre 69。 e 82。) e patológico (acima de $82 \circ)^{7}$

0 índice de retroversão, expressão quantitativa do sinal do cruzamento,8 foi registrado em ambos grupos, bem como a presença ou ausência dos sinais da espinha isquiática e da parede posterior.

Para validar os valores do ângulo alfa e do índice de retroversão no software Horos, foi medido inicialmente o coeficiente de correlação intra e interobservadores. Foram feitas duas medidas das primeiras 50 radiografias disponíveis no programa, com intervalo de 21 dias entre as avaliações. 0 coeficiente interclasse obtido foi de 0,85 para o ângulo alfa e de 0,91 para o índice de retroversão. 0 coeficiente intraclasse variou de 0,90 a 0,96 entre os examinadores para o ângulo alfa e de 0,97 a 0,98 para o índice de retroversão.

\section{Análise Estatística}

Foram utilizados os softwares SPSS V20, Minitab 16 e Excel Office 2010. Os dados descritivos foram expressos em médias, medianas e desvios-padrão. A comparação entre as variáveis foi feita com o teste t de Student pareado. O valor de p adotado foi de 0,05 e o intervalo de confiança de $95 \%$.

\section{Resultados}

O estudo incluiu 32 indivíduos (64 quadris) do sexo masculino com média de 29 anos (de 20 a 40). A prevalência de sinais radiográficos de impacto femoroacetabular com o uso como corte do ângulo alfa de 67 o foi de 53,1\% (17 controles - 28 quadris). Já com o uso como corte do valor de ângulo alfa de $82 \circ$, encontramos o valor de $31,2 \%$ ( 10 controles - 18 quadris).

0 ângulo alfa variou de 52,4 a 88,2 graus, média de 67 . Vinte e três $(35,9 \%)$ dos quadris foram considerados limítrofes, quatro $(6,3 \%)$ patológicos, corresponderam ao impacto tipo CAM. Foram considerados como limítrofe os valores entre $69 \mathrm{e}$ 82 graus e como patológicos valores maiores ou iguais a 83 graus, conforme mostrado pelo estudo de Gosvig et al. ${ }^{8}$

Quando comparada a lateralidade dos quadris para o ângulo alfa, foi observado para o lado direito uma média de 67,50 , com variação de 52,50 a 88,20 , observou-se um DP de 8,4 . No lado esquerdo, a média foi de 66,60 , variou de 53,10 a $86,90(p=0,251)$.

Foi observado um índice retroversão médio de 0,048, com valor máximo de 0,35 . Na comparação dos lados foi observada a média para o lado direito de 0,044 e para o lado esquerdo de 0,052 , com $p=0,121$.

Quando feita a comparação da distribuição do "sinal da espinha" foram identificados $15,6 \%$ de positividade, ou seja, 10 quadris. Em relação ao outro parâmetro, o "sinal da parede posterior," no entanto, foi identificado um valor ainda maior: 20,3\% de positividade, ou seja, três quadris a mais.

\section{Discussão}

O presente estudo mostrou prevalência de 31,2\% de sinais radiográficos de IFA na população assintomática estudada com o uso como valor de normalidade de corte para o ângulo alfa 82。. O valor pode ser considerado elevado, porém é menor do que o encontrado por Diesel et al. ${ }^{7}$ e Gosvig et al. ${ }^{8}$ em outros estudos, o que pode ser explicado por ter sido usado o valor de ângulo alfa considerado patológico.

A etiologia exata do IFA ainda não é bem conhecida, porém é causa comum de dor no quadril na população jovem (Samora et al. ${ }^{9}$ ) e leva potencialmente a coxartrose. Tem se observado uma maior preocupação sobre o assunto e o diagnóstico pode ser desafiador. ${ }^{4}$

Grande parte da população assintomática tem apresentado alterações radiográficas compatíveis com IFA. O valor clínico desses achados ainda é objeto de estudo, bem como a acurácia dos sinais radiográficos, especialmente para avaliação do Pincer, tem sido questionado em vários estudos. Bellaiche et al. $^{10}$ demonstraram pobre correlação do sinal do cruzamento da radiografia de frente com a versão acetabular verificada na artrorressonância. Wassilew et al. ${ }^{11}$ compararam o sinal do cruzamento e o sinal da parede posterior na radiografia com a tomografia e encontraram pior sensibilidade e especificidade nas radiografias. Por fim, Diaz-Ledesma et al. ${ }^{12}$ observaram correlação apenas entre índice de retroversão acetabular e lesão condral intraoperatória, mas não observaram tal correlação se considerados isoladamente os sinais do cruzamento, da espinha isquiática e da parede posterior. Por se tratar de estudo de prevalência em indivíduos assintomáticos, julgamos ser inapropriado solicitar tomografia computadorizada e/ou ressonância magnética por questões relativas à dose de radiação e ao custo.

\section{Conclusão}

A prevalência de sinais radiográficos numa população de homens adultos, assintomáticos e sedentários foi elevada (31,2\%). O real significado clínico desse achado ainda carece de novos estudos.

Conflitos de Interesse

Os autores declaram não haver conflitos de interesse.

\section{Referências}

1 Volpon JB. Femoroacetabular impingement. Rev Bras Ortop 2016; 51(6):621-9

2 Scheidt RB, Galia CR, Diesel CV, Rosito R, Macedo CA. Prevalence of radiographic markers of femoroacetabular impingement in asymptomatic adults. Rev Col Bras Cir 2014;41(1):36-42

3 Egger AC, Frangiamore S, Rosneck J. Femoroacetabular Impingement: a review. Sports Med Arthrosc Rev 2016;24(4):e53-8

4 Rhee C, Le Francois T, Byrd JWT, Glazebrook M, Wong I. Radiographic diagnosis of pincer-type femoroacetabular impingement: a systematic review. Orthop J Sports Med 2017;5(5): 2325967117708307

5 de Silva V, Swain M, Broderick C, McKay D. Does high level youth sports participation increase the risk of femoroacetabular impingement? A review of the current literature. Pediatr Rheumatol Online J 2016;14(1):16

6 Mascarenhas VV, Rego P, Dantas P, Morais F, McWilliams J, Collado $\mathrm{D}$, et al. Imaging prevalence of femoroacetabular impingement in 
symptomatic patients, athletes, and asymptomatic individuals: A systematic review. Eur J Radiol 2016;85(1):73-95

7 Diesel CV, Ribeiro TA, Scheidt RB, Macedo CA, Galia CR. The prevalence of femoroacetabular impingement in radiographs of asymptomatic subjects: a cross-sectional study. Hip Int 2015;25 (3):258-63

8 Gosvig KK, Jacobsen S, Palm H, Sonne-Holm S, Magnusson E. A new radiological index for assessing asphericity of the femoral head in cam impingement. J Bone Joint Surg Br 2007;89(10): 1309-16

9 Samora JB, Ng VY, Ellis TJ. Femoroacetabular impingement: a common cause of hip pain in young adults. Clin J Sport Med 2011; 21(1):51-6
10 Bellaïche L, Lequesne M, Gedouin JE, Laude F, Boyer T; French Arthroscopy Society. Imaging data in a prospective series of adult hip pain in under-50 year-olds. Orthop Traumatol Surg Res 2010; 96(8, Suppl)S44-S52

11 Wassilew GI, Heller MO, Diederichs G, Janz V, Wenzl M, Perka C. Standardized AP radiographs do not provide reliable diagnostic measures for the assessment of acetabular retroversion. J Orthop Res 2012;30(9):1369-76

12 Diaz-Ledezma C, Novack T, Marin-Peña O, Parvizi J. The relevance of the radiological signs of acetabular retroversion among patients with femoroacetabular impingement. Bone Joint J 2013;95-B(7):893-9 